\title{
A Case of Disseminated Cutaneous Mycobacterium chelonae Infection During Treatment With Tofacitinib
}

Vinit Joseph Gilvaz, MD, Chief Medical Resident, Department of Medicine, Saint Vincent Hospital; Elinor Mody, MD, Chief of Rheumatology, Reliant Medical Group, Worcester, and Department of Medicine, Saint Vincent Hospital; Saud Abaalkhail, MD, Department of Medicine, Saint Vincent Hospital, Worcester, Massachusetts, USA. Address correspondence to Dr. V.J. Gilvaz, Department of Medicine, Saint Vincent Hospital, 123 Summer Street, Worcester, MA 01608, USA. Email: Vinit.gilvaz@stvincenthospital.com. The patient's written informed consent was obtained for the publication of this material. The institutional review board at Saint Vincent Hospital and Metro West Medical Center determined that this study is not human subject research and approval is not required (IRB ID: 2020-935).

Janus kinase (JAK) inhibitors, like other immunomodulators, are known to be associated with an increased risk of infections. ${ }^{1}$ An analysis of long-term clinical trial data has shown tuberculosis to be the most common opportunistic infection associated with tofacitinib use. ${ }^{2}$ However, treatment-related nontuberculous mycobacterial (NTM) infections are exceedingly rare, and a thorough review of literature did not reveal any cases.

We present the case of a 55-year-old male who was seen in the rheumatology clinic on follow-up for seronegative rheumatoid arthritis. He had failed several prior therapies, including methotrexate, etanercept, and most recently, adalimumab, before starting tofacitinib $5 \mathrm{mg}$ twice daily, approximately 2 weeks earlier.

While having reported significant improvement in his joint symptoms, he had recently noted a new painful skin rash over his left forearm. The rash was nodular, erythematous, tender, and localized to the left forearm (Figure 1). A full-thickness skin biopsy revealed acid-fast bacilli. Of note, the patient's initial QuantiFERON-TB Gold was negative.

Over the next 2 weeks, the rash spread to involve the whole arm, with new lesions on the face. Tofacitinib was discontinued, and he was placed on a slow steroid taper instead. Final speciation by matrix-assisted laser desorption/ionization time-of-flight mass spectrometry confirmed the presence of Mycobacterium chelonae. He was subsequently started on intravenous antibiotics (tobramycin and azithromycin), which resulted in the resolution of his skin lesions.

$M$. chelonae is a rapidly growing mycobacterium that commonly presents with cutaneous disease. ${ }^{3}$ Our case highlights the need to consider NTM as a possible opportunistic infection in the setting of JAK inhibitor therapy.

\section{REFERENCES}

1. Winthrop KL. The emerging safety profile of JAK inhibitors in rheumatic disease. Nat Rev Rheumatol 2017;13:234-43.

2. Winthrop K, Park S, Gul A, Cardiel M, Gomez-Reino J, Tanaka Y, et al. Tuberculosis and other opportunistic infections in tofacitinib-treated patients with rheumatoid arthritis. Ann Rheum Dis 2016;75:1133-8.

3. Wallace Jr RJ, Brown BA, Onyi GO. Skin, soft tissue, and bone infections due to mycobacterium chelonae chelonae: importance of prior corticosteroid therapy, frequency of disseminated infections, and resistance to oral antimicrobials other than clarithromycin. J Infect Dis 1992;166:405-12.

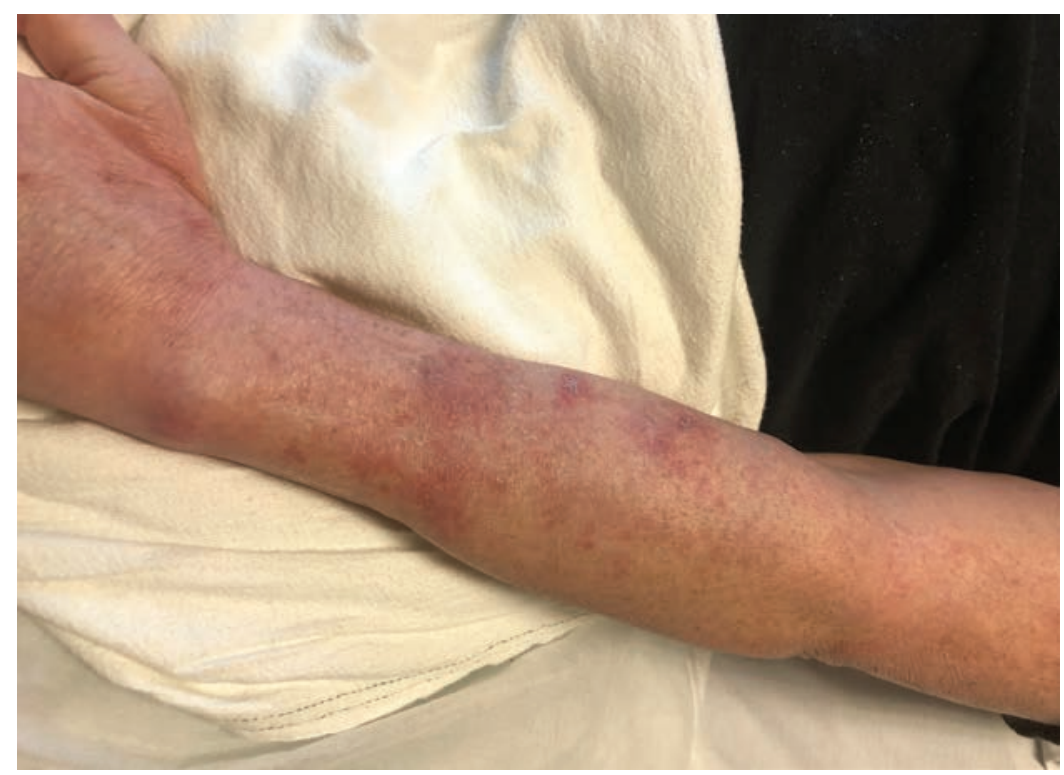

Figure 1. Mycobacterium chelonae presenting as a nodular, erythematous, tender rash, localized to the left forearm, after 2 weeks of tofacitinib treatment. 